\title{
Bağıșıklık Sistemi Etkisi Altında Tümör Büyümesinin Modellenmesi
}

\author{
Şenol KARTAL ${ }^{1, *}$ \\ ${ }^{1}$ Nevşehir Hacı Bektaş Veli Üniversitesi, Fen Edebiyat Fakültesi, Matematik Bölümü, Nevşehir
}

Özet

Bu çalışmada, tümör-bağışıklık sistemi etkileşimini tanımlamak için tam değer fonksiyonlu diferansiyel denklem sisteminden oluşan bir matematiksel model kurulmuştur. Sistem, Kuznetsov ve arkadaşlarının tümör büyümesi için önermiş olduğu matematiksel modele dayanmaktadır. Oluşturulan tam değer fonksiyonlu diferansiyel denklem sisteminin çözümünden fark denklem sistemi elde edilmiştir. Schur-Cohn kriteri ve Lyapunov fonksiyonunun kullanılmasıyla, fark denklem sisteminin pozitif denge noktasının yerel ve global kararı olmasını sağlayan yeter koşullar belirlenmiştir. Neimark-Sacker çatallanma analizi, çatallanma noktasında kararlı limit döngüsünün oluştuğu ve bunun sonucunda tümör ve bağışıklık sisteminin salınıma gittiğini göstermektedir.

Anahtar Kelimeler: Tümör -bağışıklık sistemi etkileşimi, kararlılık, fark denklem sistemi, çatallanma.

\section{Modeling a Tumor Growth under Immunological Activity}

\begin{abstract}
In this paper, a mathematical model which consists of system of differential equations with piecewise constant argument is constructed to describe tumor-immune system interaction. The system is based on the tumor growth model constructed by Kuznetsov et all. A solution of the system with piecewise constant arguments leads to a system of difference equations. Using Schur-Cohn criterion and a Lyapunov function, sufficient conditions are obtained for the local and global asymptotic stability of a positive equilibrium point of the system of difference equations. Neimark-Sacker bifurcations analysis shows that stable limit cycle occurs at the bifurcation point, thus resulting oscillations for tumor and immune system.
\end{abstract}

Keywords: Tumor -immune system interaction, stability, system of difference equation, bifurcation.

*e-mail: senol.kartal@nevsehir.edu.tr 
Kartal S.

\section{Giriş}

Matematiksel modellerin kanser biyolojisinde ve uygulanan tedavi yöntemlerinin anlaşılmasında önemli bir yeri vardır. Tümör popülasyonu ile ilgili modellemelerde denge noktasının kararlılık analizinin ve çatallanma diyagramlarının incelenmesinin hastalığın tedavisi ile ilgili çalışmalara önemli katkılar sağladığı kanıtlanmıştır. Son yıllarda bağışıklık sistemiyle kanser hücreleri arasında meydana gelen etkileşim immünoterapi (Aşı tedavisi) doğrultusunda araştırılmaya başlanmış ve immunoterapi kanser tedavisine yeni bir bakış açısı kazandırmıştır. İmmünoterapi doğal bağışıklık sisteminin güçlendirilmesi için kullanılan bir yöntemdir [1-2]. Bağışıklık sistemi hücreleri olan Sitotoksik T hücreler (CTL) tümörle mücadelede önemli rol oynayan efektör hücrelerdir. [3-4].

Birçok araştırmacı tümör-bağışıklık sistemi etkileşiminin modellenmesinde Lotka-Volterra terimler ve Verhulst (Lojistik) terimler kullanmışlardır [3-16]. Gatenby [3] tümör hücreleriyle, tümör hücrelerinin ortaya çıktığı konak hücreler arasındaki rekabeti standart Lotka-Volterra türler arası rekabet modelini kullanarak modellemiştir. Costa ve arkadaşlarının [4] oluşturduğu tümör-bağışıklık sistemi etkileşimi modeli Lotka Volterra av avcı modeline dayanmaktadır.

Kuznetsov ve arkadaşları [5] tümör hücreleriyle efektör hücreler arasındaki etkileşimi

$$
\left\{\begin{array}{l}
\frac{d E}{d t}=s+F(C, T)-d_{1} E-k_{1} E T+\left(k_{-1}+k_{2}\right) C \\
\frac{d T}{d t}=a T(1-b T)-k_{1} E T+\left(k_{-1}+k_{3}\right) C \\
\frac{d C}{d t}=k_{1} E T-\left(k_{-1}+k_{2}+k_{3}\right) C \\
\frac{d E^{*}}{d t}=k_{3} C-d_{2} E^{*} \\
\frac{d T^{*}}{d t}=k_{2} C-d_{3} T^{*}
\end{array}\right.
$$

modeliyle tanımlamıştır. Burada E efektör hücre (EC) konsantrasyonunu, T tümör hücre (TC) konsantrasyonunu, C etkileşimde olan efektör hücre-tümör hücre konsantrasyonunu, $\mathrm{E}^{*}$ aktif olmayan efektör hücreleri, $\mathrm{T}^{*}$ öldürülmeye programlanmış tümör hücrelerini temsil eder. $\mathrm{k}_{1}$, efektör hücrelerin tümör hücrelerine bağlanma oranı, $\mathrm{k}_{-1}$ efektör hücre-tümör hücre etkileşimi sonucunda efektör hücrelerinin zarar görmeden ayrılma oranı, $\mathrm{k}_{2}$ EC-TC etkileşimi sonucunda TC hücrelerin programlanmış bir şekilde geri dönüşümsüz olarak kendini sindirme oranı, $\mathrm{k}_{3}$ EC-TC etkileşimi sonucunda EC hücrelerin inaktif olma oranıdır. s olgun EC hücrelerinin tümör yerlerine akış oranı, $\mathrm{d}_{1} \mathrm{E}$ nin yok edilme oranı, $\mathrm{d}_{2}$ $\mathrm{E}^{*}$ nin yok edilme oranı, $\mathrm{d}_{3} \mathrm{~T}^{*}$ nin yok edilme oranı, $a$ tümörün maksimum büyüme oranı, $\mathrm{b}^{-1}$ tümör maksimum taşıma kapasitesidir. Efektör hücrelerin tümör bölgesine birikme oranı $F(C, T)=\frac{f C}{g+T}$ fonksiyonu ile tanımlanır. Burada f ve g pozitif sabitlerdir. Çalışmada bir eşik değeri tahmin edilmiştir ki bu eşik değerin üstünde kanser hücreleri kontrolsüz bir şekilde büyürken, eşik değerin altında ise hastalığ1 oluşturan tümör popülasyonunda 3-4 ayda bir periyodik dalgalanmalar görülmesine rağmen genel olarak popülasyonda azalma gözlenmiştir [5].

Galach [6] ve Yafia [7] (1) modeli için $\frac{\mathrm{dC}}{\mathrm{dt}} \approx 0$ yaklaşımında bulunarak $\mathrm{C} \approx$ KET olduğunu varsaymışlardır. Burada $\mathrm{K}=\mathrm{k}_{1} /\left(\mathrm{k}_{2}+\mathrm{k}_{3}+\mathrm{k}_{-1}\right)$ dır. $\alpha_{1}=\theta-\mathrm{m}, \mathrm{n}=\mathrm{K} / \mathrm{k}_{2}, \mathrm{~m}=\mathrm{K} / \mathrm{k}_{3}, \mathrm{~d}=\mathrm{d}_{1}$ ve $\mathrm{F}(\mathrm{C}, \mathrm{T})=\mathrm{F}(\mathrm{E}, \mathrm{T})=\theta \mathrm{ET}$ olmak üzere (1) sistemi 
$\left\{\begin{array}{l}\frac{d E}{d t}=s+\alpha_{1} E T-d E \\ \frac{d T}{d t}=a T(1-b T)-n E T\end{array}\right.$

şeklinde yazılabilir. (2) sisteminin boyutsuzlaştırılmasıyla

$\left\{\frac{d x}{d t}=\sigma+\omega x y-\delta x\right.$

$\left\{\frac{d y}{d t}=\alpha y(1-\beta y)-x y\right.$

sistemi elde edilir. Burada $\alpha=\mathrm{a} / \mathrm{Kk}_{2} \mathrm{~T}_{0}, \beta=\mathrm{bT}, \delta=\mathrm{d} / \mathrm{Kk}_{2} \mathrm{~T}_{0}, \sigma=\mathrm{s} / \mathrm{nE}_{0} \mathrm{~T}_{0}$ ve $\omega=\alpha_{1} \mathrm{n} \operatorname{dir}$ [6].

Kirschner ve Panetta [8] Kuznetsov'un modelini genelleştirerek sitokinlerin tümör dinamiği üzerindeki etkisini araştırmışlardır. Pillis ve arkadaşları [9] önerdikleri tümör bağışıklık etkileşimi modelinde NK hücrelerin ve sitotoksik T hücrelerin rolünü araştırmışlardır. Sarkar ve Banerjee [10], malignant (kötü huylu tümör) hücrelerle bağışıklık sistemi hücreleri arasındaki etkileşimi av-avcı sistemine benzer bir sistemle modellemişlerdir.

Tümör hücrelerinin çoğalması, aktivasyonu ve bağışıklık sistemi hücreleriyle rekabeti mikroskobik düzeyde gerçekleşen biyolojik olaylar iken, kanser yayılımı ve metastazı makroskobik düzeyde gerçekleşen biyolojik olaylardır. Mikroskobik düzeyde gerçekleşen biyolojik olaylar süreklilikten ziyade kesiklilik (discrete) arz ettiği için, birçok araştırmacı tümör bağışıklık sistemi etkileşimi için kesikli zamanlı dinamik modeller oluşturmuşlardır [17-21]. Diğer yandan tümör büyümesini daha iyi anlamak için hem mikroskobik düzeyde gerçekleşen etkileşimleri hem de makroskobik düzeyde oluşan olayları aynı anda göz önüne alan matematiksel modellerin oluşturulması gereklidir [17]. Hem mikroskobik hem de makroskobik düzeyde gerçekleşen biyolojik olaylar aynı anda göz önüne alınırsa tümör popülasyonu için iki durum söz konusudur: tümör popülasyonunda süreklilik ve tümör hücreleri için dinlenme evresi. Her iki durum için, süreklilik ve kesiklilik, ekosistemde hem diferansiyel hem de fark denklemlerin özelliklerinin birlikte yer aldığı bazı popülasyon dinamikleri vardır. Bu gibi biyolojik olaylar için birçok araştırmacı sabit katsayılı tam değer fonksiyonlu diferansiyel denklemler kullanmışlardır [22-32].

Bu çalışmada (3) sistemine parçalı tam değer fonksiyonu $(\llbracket t \rrbracket, t \in(0, \infty])$ katılarak oluşturulan

$\left\{\begin{array}{l}\frac{d x}{d t}=\sigma+\omega x(t) y(\llbracket t \rrbracket)-\delta x(t) \\ \frac{d y}{d t}=\alpha y(t)(1-\beta y(t))-x(\llbracket t \rrbracket) y(t)\end{array}\right.$

sistemin yerel ve global davranışları incelenecektir. Burada $\mathrm{x}(\mathrm{t})$ efektör hücre popülasyonunu, $\mathrm{y}(\mathrm{t})$ tümör hücre popülasyonunu, $t$ zamanı ve $\llbracket t \rrbracket, t \in[0, \infty)$ için t'nin tam değerini göstermektedir. $\sigma$ efektör hücrelerin tümör bölgesine akış oranı, $\omega$ bağışıklık cevabın tümör hücreleri üzerindeki etkisi, $\delta$ efektör hücrelerin ölüm oranı, $\alpha$ tümör büyüme oranı ve $\beta^{-1}$ tümör maksimum taşıma kapasitesidir.

\section{Yerel ve Global Kararlılık Analizi}

(4) sistemi t $\epsilon[n, n+1)$ aralığında 


$$
\left\{\begin{array}{l}
\frac{d x}{d t}+x(t)(\delta-\omega y(n))=\sigma \\
\frac{d y}{d t}-y(t)(\alpha-x(n))=-\alpha \beta y^{2}(t)
\end{array}\right.
$$

şeklinde yazılabilir. (5) sistemindeki her bir denklem [n,t) aralığında $t$ ye integre edilip $t \rightarrow n+1$ alınırsa

$$
\left\{\begin{array}{l}
\mathrm{x}(\mathrm{n}+1)=\mathrm{x}(\mathrm{n}) \mathrm{e}^{-\delta+\omega \mathrm{y}(\mathrm{n})}+\left(\frac{\sigma\left(1-\mathrm{e}^{-\delta+\omega \mathrm{y}(\mathrm{n})}\right)}{\delta-\omega \mathrm{y}(\mathrm{n})}\right) \\
\mathrm{y}(\mathrm{n}+1)=\frac{y(n)[\alpha-\mathrm{x}(\mathrm{n})]}{[\alpha-\mathrm{x}(\mathrm{n})-\alpha \beta y(n)] \mathrm{e}^{-[\alpha-\mathrm{x}(\mathrm{n})]}+\alpha \beta y(n)}
\end{array}\right.
$$

fark denklem sistemi elde edilir. (6) fark denklem sisteminin global davranışlarını analiz etmek için denge noktasına ihtiyaç vardır.

$\alpha \delta>\sigma$

koşulu altında (6) sisteminin pozitif denge noktası

$(\overline{\mathrm{x}}, \overline{\mathrm{y}})=\left(\frac{\alpha(-\beta \delta+\omega)+\sqrt{\Delta}}{2 \omega}, \frac{\alpha(\beta \delta+\omega)-\sqrt{\Delta}}{2 \alpha \beta \omega}\right)$

olarak hesaplanır. Burada $\Delta=4 \beta \sigma \omega \alpha+\alpha^{2}(-\beta \delta+\omega)^{2}$ dır. (6) sisteminin pozitif denge noktasıyla (3) sisteminin kritik noktası aynıdır.

$(\bar{x}, \bar{y})$ denge noktası civarında (6) fark denklem sisteminin lineerleştirilmiş sistemi $w(n+1)=$ Aw(n) olsun. Burada A matrisi

$$
A=\left(\begin{array}{cc}
\mathrm{e}^{\frac{-\alpha(\beta \delta-\omega)-\sqrt{\Delta}}{2 \alpha \beta}} & \frac{4 \alpha^{2} \beta^{2} \sigma \omega\left(1-\mathrm{e}^{\frac{-\alpha(\beta \delta-\omega)-\sqrt{\Delta}}{2 \alpha \beta}}\right)}{(\alpha(\beta \delta-\omega)+\sqrt{\Delta})^{2}} \\
-\frac{1-\mathrm{e}^{\frac{-\alpha(\beta \delta+\omega)+\sqrt{\Delta}}{2 \omega}}}{\alpha \beta} & \mathrm{e}^{\frac{-\alpha(\beta \delta+\omega)+\sqrt{\Delta}}{2 \omega}}
\end{array}\right)
$$

şeklindedir. A matrisinin karakteristik denklemi ise

$$
\begin{aligned}
\mathrm{p}(\lambda)= & \lambda^{2}+\lambda\left(-\mathrm{e}^{\frac{-\alpha(\beta \delta-\omega)-\sqrt{\Delta}}{2 \alpha \beta}}-\mathrm{e}^{\frac{-\alpha(\beta \delta+\omega)+\sqrt{\Delta}}{2 \omega}}\right)+\mathrm{e}^{\frac{-\alpha(\beta \delta-\omega)-\sqrt{\Delta}}{2 \alpha \beta}} \mathrm{e}^{\frac{-\alpha(\beta \delta+\omega)+\sqrt{\Delta}}{2 \omega}} \\
& \left.+\frac{4 \alpha^{2} \beta^{2} \sigma \omega\left(1-\mathrm{e}^{\frac{-\alpha(\beta \delta-\omega)-\sqrt{\Delta}}{2 \alpha \beta}}\right)\left(\frac{1-\mathrm{e}^{\frac{-\alpha(\beta \delta+\omega)+\sqrt{\Delta}}{2 \omega}}}{\alpha \beta}\right)}{(\alpha(\beta \delta-\omega)+\sqrt{\Delta})^{2}}\right)
\end{aligned}
$$

şeklinde hesaplanır. (6) denklem sisteminin pozitif denge noktasının yerel kararlılık koşullarını $p(\lambda)$ denklemi boyunca elde etmek için aşağıdaki teoremde verilen Schur-Chon kriteri kullanılabilir.

Teorem 1. ([33]) $p(\lambda)=\lambda^{2}+p_{1} \lambda+p_{0}$

karakteristik polinomunun tüm kökleri birim dairenin içindedir ancak ve ancak
(a) $\mathrm{p}(1)=1+\mathrm{p}_{1}+\mathrm{p}_{0}>0$
(b) $\mathrm{p}(-1)=1-\mathrm{p}_{1}+\mathrm{p}_{0}>0$
(c) $\mathrm{D}_{1}^{+}=1+\mathrm{p}_{0}>0$
(d) $\mathrm{D}_{1}^{-}=1-\mathrm{p}_{0}>0$

ise.

Teorem 2. $(\bar{x}, \bar{y}),(6)$ fark denklem sisteminin pozitif denge noktası ve 
$\frac{\sigma}{\alpha}<\delta$

olsun. Ĕger

$\sigma<\frac{(\alpha(\beta \delta-\omega)+\sqrt{\Delta})(4 \alpha \beta \omega+(\sqrt{\Delta}+\alpha(\beta(-2+\delta)-\omega))(\sqrt{\Delta}+2 \omega-\alpha(\beta \delta+\omega)))}{4 \alpha \beta \omega(-\sqrt{\Delta}+\alpha(\beta \delta+\omega))}$

ise $(\bar{x}, \bar{y})$ denge noktası yerel asimptotik kararlıdır.

İspat. Teorem 1 in koşullarını analiz edelim. (a) dan

$$
\begin{aligned}
\mathrm{p}(1)= & \frac{4 \alpha^{2} \beta^{2} \sigma \omega\left(1-\mathrm{e}^{\frac{-\alpha(\beta \delta-\omega)-\sqrt{\Delta}}{2 \alpha \beta}}\right)}{(\alpha(\beta \delta-\omega)+\sqrt{\Delta})^{2}}\left(\frac{1-\mathrm{e}^{\frac{-\alpha(\beta \delta+\omega)+\sqrt{\Delta}}{2 \omega}}}{\alpha \beta}\right) \\
& +\left(1-\mathrm{e}^{\frac{-\alpha(\beta \delta-\omega)-\sqrt{\Delta}}{2 \alpha \beta}}\right)\left(1-\mathrm{e}^{\frac{-\alpha(\beta \delta+\omega)+\sqrt{\Delta}}{2 \omega}}\right)>0
\end{aligned}
$$

elde edilir. (11) eşitsizliği

$-\alpha(\beta \delta-\omega)-\sqrt{\Delta}<0$

ve

$-\alpha(\beta \delta+\omega)+\sqrt{\Delta}<0$

koşulları altında mevcuttur. (12) ve (13) eşitsizlikleri ise (7) koşulu altında her zaman doğrudur. (b) den

$\mathrm{p}(-1)=1+\mathrm{e}^{\frac{-\alpha(\beta \delta-\omega)-\sqrt{\Delta}}{2 \alpha \beta}} \mathrm{e}^{\frac{-\alpha(\beta \delta+\omega)+\sqrt{\Delta}}{2 \omega}}$

$$
+\frac{4 \alpha^{2} \beta^{2} \sigma \omega\left(1-\mathrm{e}^{\frac{-\alpha(\beta \delta-\omega)-\sqrt{\Delta}}{2 \alpha \beta}}\right)}{(\alpha(\beta \delta-\omega)+\sqrt{\Delta})^{2}}\left(\frac{1-\mathrm{e}^{\frac{-\alpha(\beta \delta+\omega)+\sqrt{\Delta}}{2 \omega}}}{\alpha \beta}\right)+\mathrm{e}^{\frac{-\alpha(\beta \delta-\omega)-\sqrt{\Delta}}{2 \alpha \beta}}+\mathrm{e}^{\frac{-\alpha(\beta \delta+\omega)+\sqrt{\Delta}}{2 \omega}}>0
$$

yazılabilir. Yine (12) ve (13) koşulları için $\mathrm{p}(-1)>0$ olduğu açıktır. (c) den

$\mathrm{D}_{1}^{+}=1+\mathrm{e}^{\frac{-\alpha(\beta \delta-\omega)-\sqrt{\Delta}}{2 \alpha \beta}} \mathrm{e}^{\frac{-\alpha(\beta \delta+\omega)+\sqrt{\Delta}}{2 \omega}}+\frac{4 \alpha^{2} \beta^{2} \sigma \omega\left(1-\mathrm{e}^{\frac{-\alpha(\beta \delta-\omega)-\sqrt{\Delta}}{2 \alpha \beta}}\right)}{(\alpha(\beta \delta-\omega)+\sqrt{\Delta})^{2}}\left(\frac{1-\mathrm{e}^{\frac{-\alpha(\beta \delta+\omega)+\sqrt{\Delta}}{2 \omega}}}{\alpha \beta}\right)>0$

bulunur ki bu eşitsizlik de her zaman mevcuttur. (d) den

$\mathrm{D}_{1}^{-}=\mathrm{e}^{\frac{-\alpha(\beta \delta-\omega)-\sqrt{\Delta}}{2 \alpha \beta}} \mathrm{e}^{\frac{-\alpha(\beta \delta+\omega)+\sqrt{\Delta}}{2 \omega}}+\frac{4 \alpha^{2} \beta^{2} \sigma \omega\left(1-\mathrm{e}^{\frac{-\alpha(\beta \delta-\omega)-\sqrt{\Delta}}{2 \alpha \beta}}\right)\left(1-\mathrm{e}^{\frac{-\alpha(\beta \delta+\omega)+\sqrt{\Delta}}{2 \omega}}\right)}{\alpha \beta(\alpha(\beta \delta-\omega)+\sqrt{\Delta})^{2}}<1$

elde edilir. $\mathrm{e}^{\frac{-\alpha(\beta \delta-\omega)-\sqrt{\Delta}}{2 \alpha \beta}}$ ve $\mathrm{e}^{\frac{-\alpha(\beta \delta+\omega)+\sqrt{\Delta}}{2 \omega}}$ ifadeleri sirasiyla $0<\frac{\alpha(\beta \delta-\omega)+\sqrt{\Delta}}{2 \alpha \beta}<1$ ve $0<\frac{\alpha(\beta \delta+\omega)-\sqrt{\Delta}}{2 \omega}<1$

aralığında seriye açılırsa

$\mathrm{e}^{-\frac{\alpha(\beta \delta-\omega)+\sqrt{\Delta}}{2 \alpha \beta}}=1-\frac{\alpha(\beta \delta-\omega)+\sqrt{\Delta}}{2 \alpha \beta}$

ve

$\mathrm{e}^{-\frac{\alpha(\beta \delta+\omega)-\sqrt{\Delta}}{2 \omega}}=1-\frac{\alpha(\beta \delta+\omega)-\sqrt{\Delta}}{2 \omega}$

yazılabilir. Bu ifadeler $\mathrm{D}_{1}^{-}$eşitsizliğinde yerine yazılıp eşitsizlik yeniden düzenlenirse 


\section{Kartal S.}

$\sigma<\frac{(\alpha(\beta \delta-\omega)+\sqrt{\Delta})(4 \alpha \beta \omega+(\sqrt{\Delta}+\alpha(\beta(-2+\delta)-\omega))(\sqrt{\Delta}+2 \omega-\alpha(\beta \delta+\omega)))}{4 \alpha \beta \omega(-\sqrt{\Delta}+\alpha(\beta \delta+\omega))}$

elde edilir.

Örnek 1. [7] çalışmasındaki $\sigma=0.1181, \beta=0.002, \delta=0.3747, \omega=0.01184$ parametre değerleri ve $\alpha=0.7$ değeri Teorem 2 in koşullarını sağlamaktadır. Bu parametre değerleri ve $\mathrm{x}(1)=2, \mathrm{y}(1)=2$ başlangıç koşulları için $(\bar{x}, \bar{y})=(0.676341,16.899)$ denge noktasının yerel asimptotik kararlı olduğu Şekilı den görülebilir. Burada mavi renk x(n) (efektör hücre popülasyonunu), kırmızı renk y(n) (tümör hücre popülasyonunu) temsil eder.

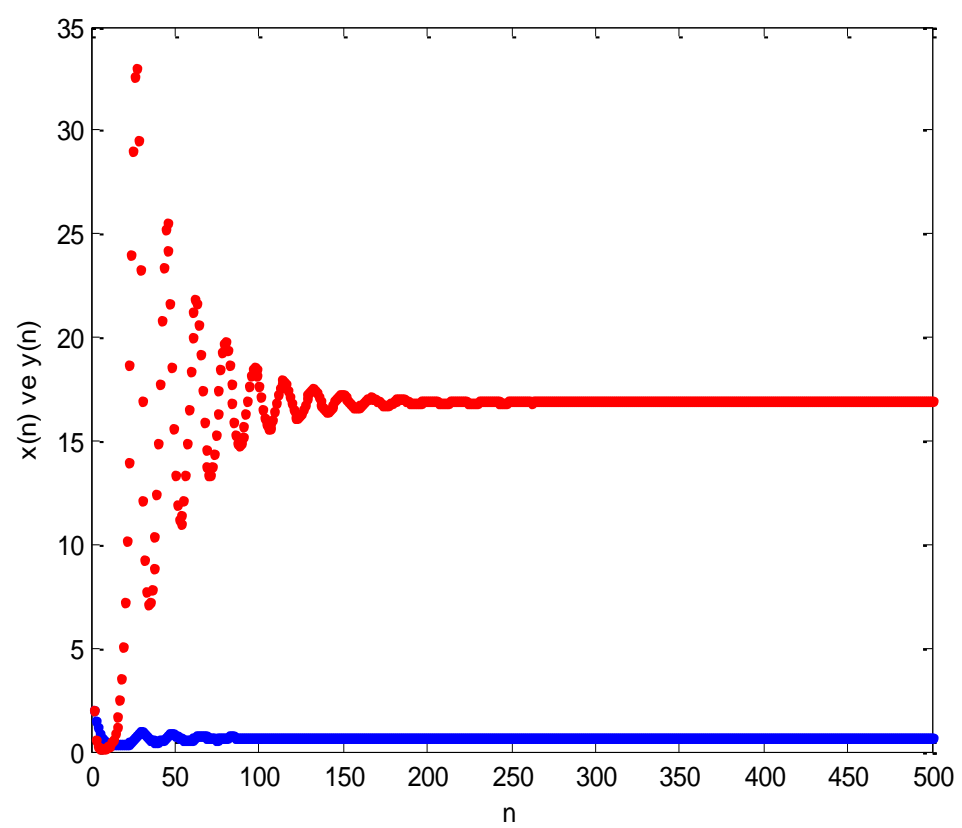

Şekil 1 x(n) ve y(n) çözümlerinin iterasyon grafikleri.

Teorem 3. Teorem 2 in koşullarının mevcut olduğunu ve

$\delta-\omega \mathrm{y}(\mathrm{n})>0, \quad \alpha-\mathrm{x}(\mathrm{n})>0, \quad \mathrm{x}(\mathrm{n})>2 \bar{x} \quad$ ve $\quad \mathrm{y}(\mathrm{n})<\overline{\mathrm{y}}$.

olduğunu varsayalım. Eğer

$\mathrm{x}(\mathrm{n})>\frac{\sigma}{\delta-\omega \mathrm{y}(\mathrm{n})}$

$\alpha \beta y(n)<\alpha-x(n)<\ln \left[\frac{2 \bar{y}-y(n)}{y(n)}\right]$

ise (6) sistemin pozitif denge noktası global asimptotik kararlıdır.

İspat. $\bar{E}=(\bar{x}, \bar{y})(6)$ sisteminin pozitif denge noktası olsun. $V(n)$

$\mathrm{V}(\mathrm{n})=[\mathrm{E}(\mathrm{n})-\overline{\mathrm{E}}]^{2}, \mathrm{n}=0,1,2 \ldots$

şeklinde bir Lyapunov fonksiyonu tanımlayalım. (6) sistemine fark operatörü uygulanırsa

$\Delta \mathrm{V}(\mathrm{n})=\mathrm{V}(\mathrm{n}+1)-\mathrm{V}(\mathrm{n})$

$$
=\{E(n+1)-E(n)\}\{E(n+1)+E(n)-2 \bar{E}\} .
$$


elde edilir. (6) sistemindeki birinci denklemden

$$
\begin{aligned}
\Delta \mathrm{V}_{1}(\mathrm{n}) & =[\mathrm{x}(\mathrm{n}+1)-\mathrm{x}(\mathrm{n})][\mathrm{x}(\mathrm{n}+1)+\mathrm{x}(\mathrm{n})-2 \overline{\mathrm{x}}] \\
& =\frac{\left(1-\mathrm{e}^{-\mathrm{A}_{1}}\right)\left(\sigma-\mathrm{A}_{1} \mathrm{x}(\mathrm{n})\right)}{\mathrm{A}_{1}} \frac{\mathrm{A}_{1} \mathrm{x}(\mathrm{n}) \mathrm{e}^{-\mathrm{A}_{1}}+\sigma\left(1-\mathrm{e}^{-\mathrm{A}_{1}}\right)+\mathrm{A}_{1}(\mathrm{x}(\mathrm{n})-2 \bar{x})}{\mathrm{A}_{1}}
\end{aligned}
$$

yazılabilir. Burada $A_{1}=\delta-\omega y(n)>0$ dır. (14) eşitsizliği ve $x(n)>2 \bar{x}$ eşitsizliği göz önüne alınırsa, $\Delta \mathrm{V}_{1}(\mathrm{n})<0$ elde edilir.

Benzer şekilde (15) eşitsizliğinin varlığg altında $\Delta \mathrm{V}_{2}(\mathrm{n})=[\mathrm{y}(\mathrm{n}+1)-\mathrm{y}(\mathrm{n})][\mathrm{y}(\mathrm{n}+1)+\mathrm{y}(\mathrm{n})-2 \overline{\mathrm{y}}]<0$ olduğu görülebilir. Sonuç olarak $\Delta \mathrm{V}(\mathrm{n})=\left(\Delta \mathrm{V}_{1}(\mathrm{n}), \Delta \mathrm{V}_{2}(\mathrm{n})\right)<0$ sonucuna ulaşılır.

\section{Neimark-Sacker Çatallanma Analizi}

Neimark-Sacker çatallanması, kesikli zamanlı dinamik modellerde ortaya çıkan oldukça önemli bir çatallanmadır. Çünkü bu çatallanma sonucunda bir limit döngüsü (limit cycle) oluşur ki bu da periyodik çözümlerin varlığına işaret eder. Bu çatallanma tipi için linerleştirilmiş sistemin jakobyen matrisinin karakteristik denklemi birim dairenin üzerinde olan bir çift eşlenik kompleks özdeğere sahiptir ve karakteristik denklemin diğer bütün özdeğerleri birim dairenin içindedir. Aşağıdaki teorem (SchurCohn kriteri) 2. mertebeden bir karakteristik denkleme sahip fark denklem sisteminde Neimark-Sacker çatallanmasının olabilmesi için gerek ve yeter cebirsel koşulları vermektedir.

Teorem 4 ([33])

$\mathrm{p}(\lambda)=\lambda^{2}+\mathrm{p}_{1} \lambda+\mathrm{p}_{0}$

denkleminin bir çift eşlenik kompleks kökü birim dairenin üzerinde ve $p(\lambda)$ denkleminin diğer bütün kökleri birim dairenin içindedir ancak ve ancak

(a) $\mathrm{p}(1)=1+\mathrm{p}_{1}+\mathrm{p}_{0}>0$

(b) $\mathrm{p}(-1)=1-\mathrm{p}_{1}+\mathrm{p}_{0}>0$

(c) $\mathrm{D}_{1}^{+}=1+\mathrm{p}_{0}>0$

(d) $\mathrm{D}_{1}^{-}=1-\mathrm{p}_{0}=0$

(6) sisteminin pozitif denge noktasının kararlılıktan kararsızlığa geçiş noktası olan çatallanma noktası Teorem 4 deki koşulların analiz edilmesiyle aşağıdaki örnekte olduğu gibi belirlenebilir.

Örnek 2. Yerel kararlılık analizinden $\mathrm{p}(1)>0 \mathrm{p}(-1)>0$ ve $\mathrm{D}_{1}^{+}>0$ eşitsizliklerinin her zaman mevcut olduğunu biliyoruz. Teorem 4/(d), deki denklem $\alpha$ ya gore çözülürse $\bar{\alpha}=0.82747$ elde edilir. Yine Örnek 1 deki parametre değerleri ve $\bar{\alpha}=0.82747$ için sistemin karakteristik denkleminden özdeğerlerin normları $\left|\lambda_{1,2}\right|=|0.915476 \pm 0.402374 \mathrm{i}|=1$ şeklinde hesaplanır. Şekil 2, sistemin çözümlerinin $\bar{\alpha}=0.82747$ noktasında çatallandığını göstermektedir. Burada $\mathrm{x}(1)=2, \mathrm{y}(1)=2$ olarak alınmıştır. 

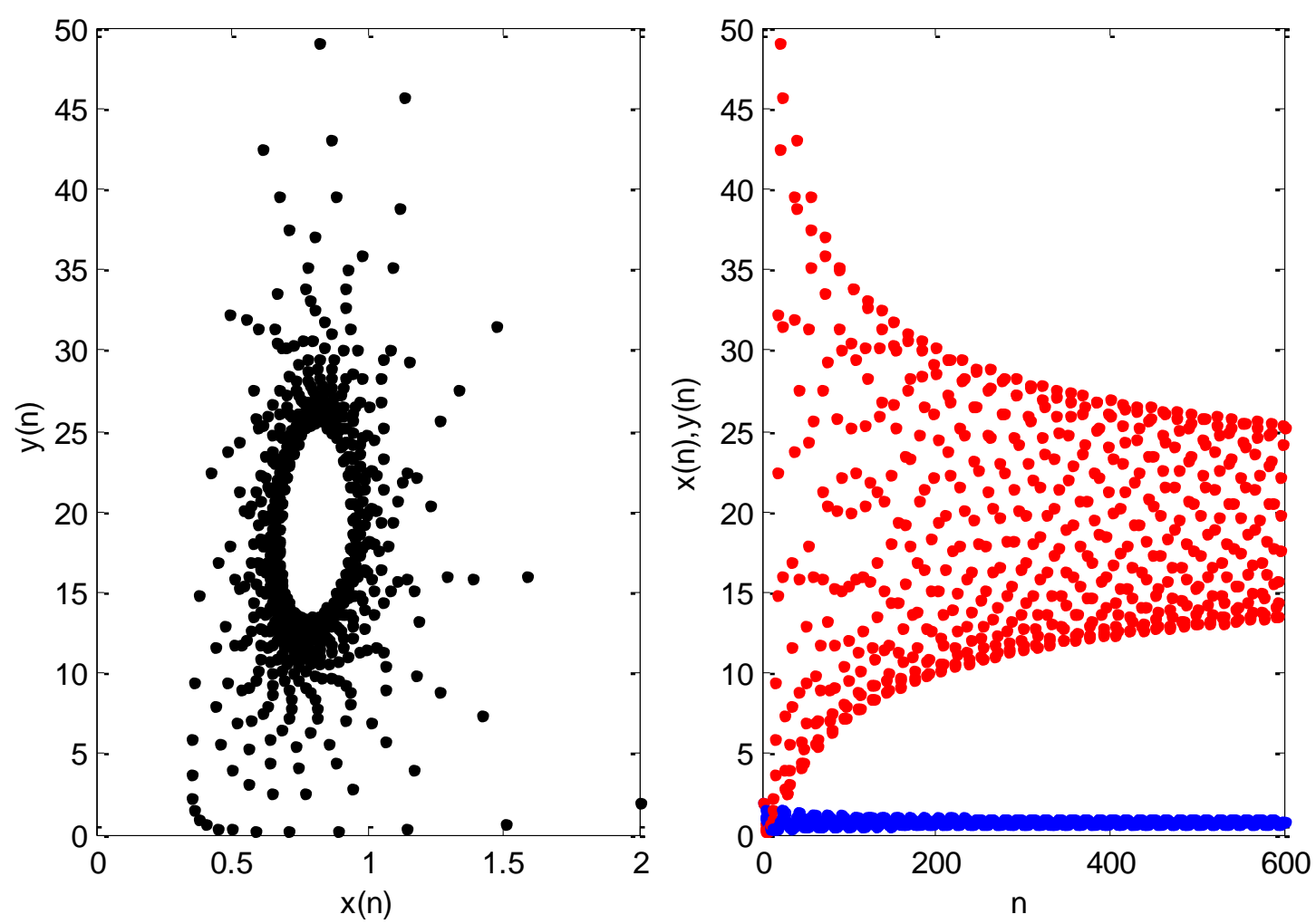

Şekil $2 \bar{\alpha}=0.82747$ değeri için sistemin Neimark-Sacker çatallanması.

Sonuç olarak eğer $0<\alpha<0.82747$ ise sistemin pozitif denge noktası kararlı aksi takdirde kararsızdır (Şekil 3 ve Şekil 4).

Örnek 3. $\alpha=1$ değeri için (10) karakteristik denkleminden özdeğerler ve bu özdeğerlerin normları (diğer parametre değerleri Örnek 1 deki gibidir) $\left|\lambda_{1,2}\right|=|0.921197 \pm 0.469375 i|=1.03388>1$ şeklinde elde edilir. Şekil 3 sistemin pozitif denge noktasının $\alpha=1$ değeri için kararsız olduğunu göstermektedir. Burada mavi renk x(n) efektör hücre popülasyonunu, kırmızı renk y(n) tümör hücre popülasyonunu temsil eder. 

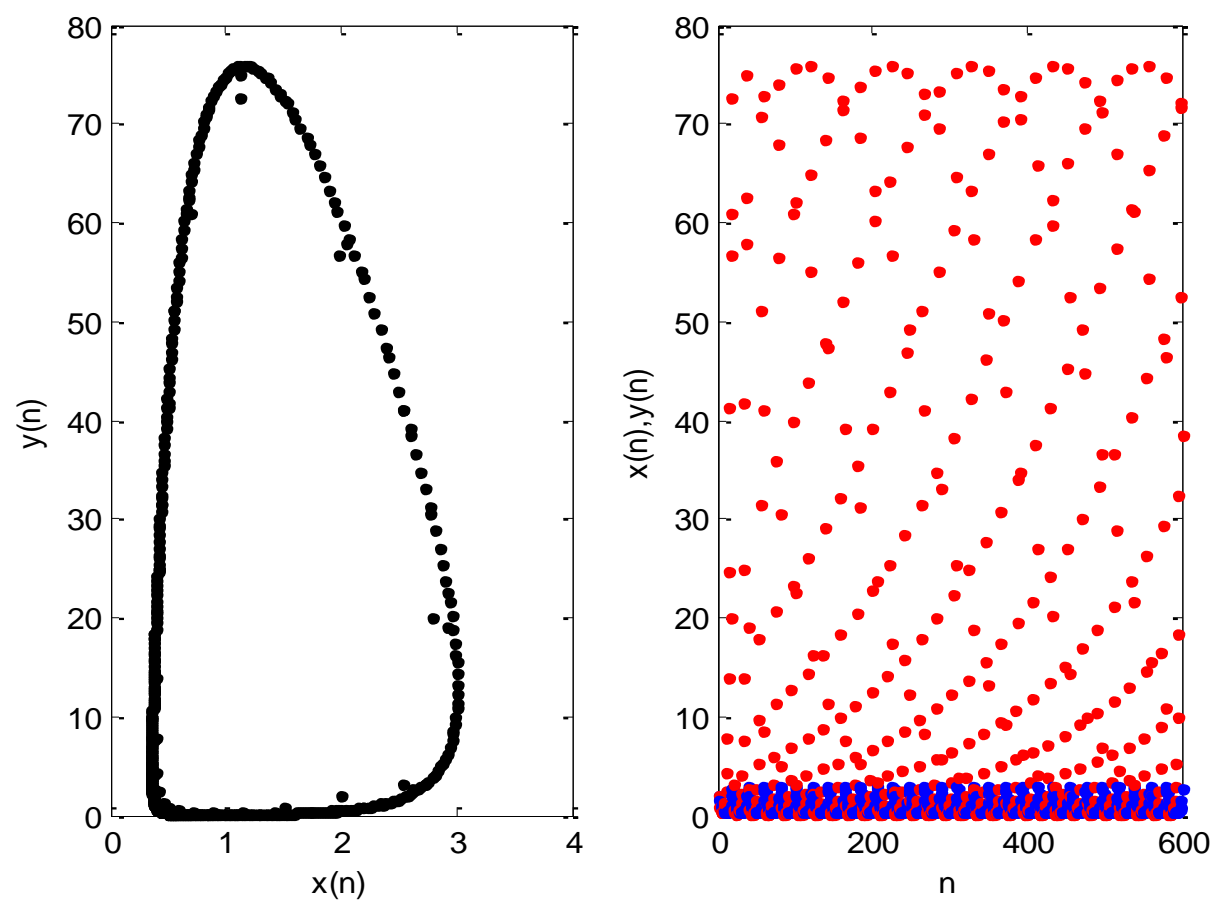

Şekil $3 \alpha=1$ değeri ve $\mathrm{x}(1)=2, \mathrm{y}(1)=2$ başlangıç koşulları için sistemin iterasyon grafiği .

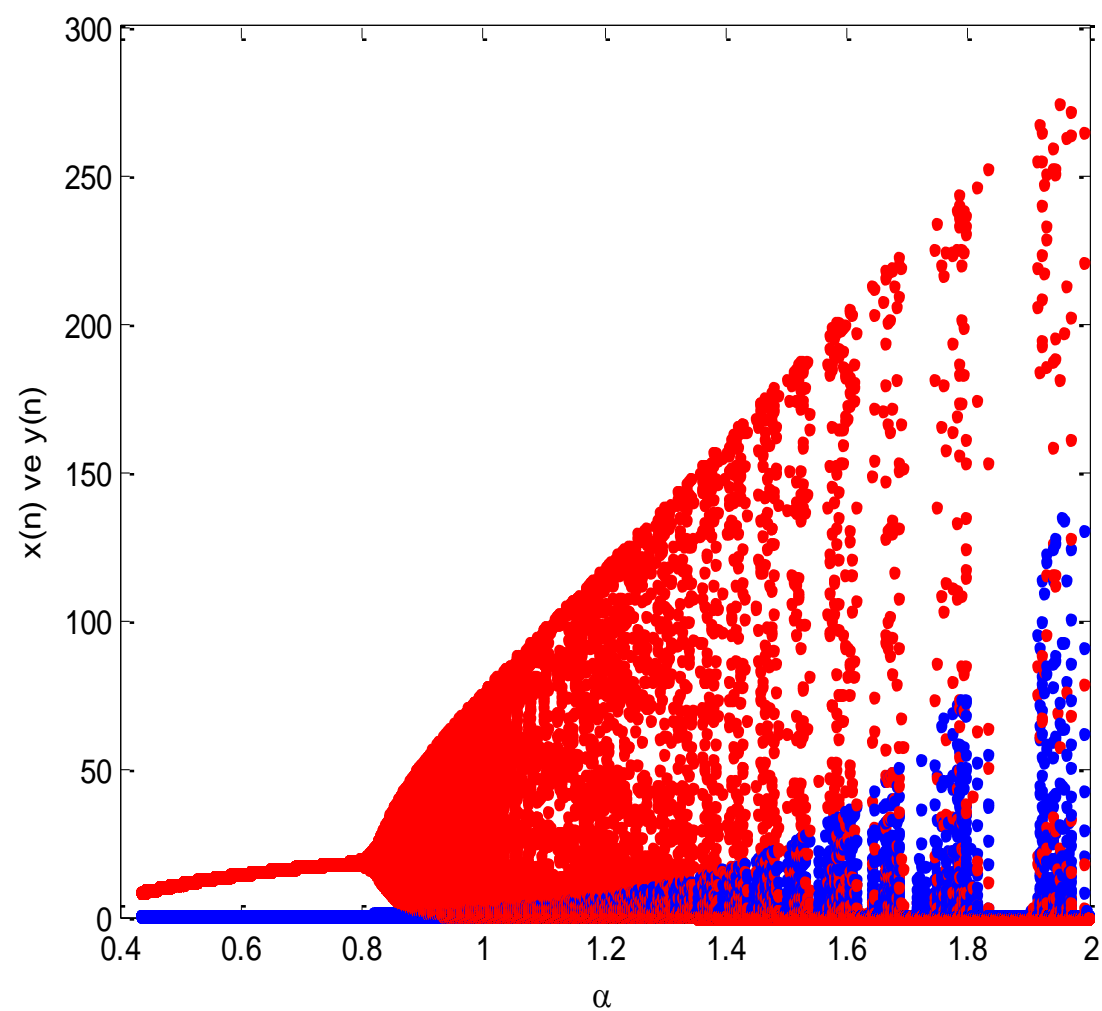

Şekil $4 \alpha \in[0.436,2]$ ve $x(1)=2, y(1)=2$ başlangıç koşulları için $\alpha$ ya karşı çizilmiş (6) sisteminin $x(n)$ ve $y(n)$ popülasyonlarının çatallanması.

Teşekkür. Bu çalışmaya katkılarından dolayı değerli hocam Prof. Dr. Fuat Gürcan'a teşekkür ederim. 


\section{Kaynaklar}

[1] Pillis L. G. De., Gu W., Radunskaya A. E., " Mixed immunotherapy and chemotherapy of tumors: modeling applications and biological interpretations" J. Theor. Biol., 238 (4), 841-862, 2006.

[2] Ghaffari A., Naserifar, N., " Optimal therapeutic protocols in cancer immunotherapy" Comput. Biol. Med., 40, 261-270, 2010.

[3] Gatenby R. A., "Models of tumor-host interaction as competing populations: implications for tumor biology and treatment" J. Theor. Biol., 176, 447-455, 1995.

[4] Costa O. S., Molina L. M., Perez D. R., Antoranz J. C., Reyes M. C., "Behavior of tumors under nonstationary therapy" Physica D, 178, 242-253, 2003.

[5] Kuznetsov, V. A., Makalkin I. A., Taylor, M. A., Perelson, A. S, "Nonlinear dynamics of immunogenic tumors: parameter estimation and global bifurcation analysis" Bull. Math. Biol., 56, 295-321, 1994.

[6] Galach, M., "Dynamics of the tumor-immune system competition-the effect of time delay" Int. J. Appl. Math. Comput. Sci., 13, 395-406, 2003.

[7] Yafia, R., "Hopf bifurcation analysis and numerical simulations in an ODE model of the immune system with positive immune response" Nonlinear Anal. Real., 8, 1359-1369, 2007.

[8] Kirschner D., Panetta J.C., "Modeling immunotherapy of the tumor-immune interaction" $J$. Math. Biol., 37, 235-252, 1998.

[9] Pillis L. G. De, Radunskaya A., "A mathematical tumor model with immune resistance and drug therapy: an optimal control approach" J. Theor. Med,. 3, 79-100, 2001.

[10] Sarkar R. R., Banerjee S., "Cancer self remission and tumor stability- a stochastic approach" Math. Biosci., 196, 65-81, 2005.

[11] Merola A., Cosentino C., Amato F., "An insight into tumor dormancy equilibrium via the analysis of its domain of attraction" Bio. Sig. Proc. Control., 3, 212-219, 2008.

[12] Onofrio A. D., "Metamodeling tumor-immune system interaction, tumor evasion and immunotherapy" Math. Comput. Model., 47, 614-637, 2008.

[13] Baker C. T. H., Bocharov G.A., Paul C. A. H., "Mathematical modeling of the interleukin-2 Tcell system: a comparative study of approaches based on ordinary and delay differential equations" J. Theor. Med., 2, 117-128, 1997.

[14] Banerjee S., "Immunotherapy with interleukin-2: a study based on mathematical modeling" Int. J. Appl. Math. Comput. Sci., 18, 389-398, 2008.

[15] Villasana M., Radunskaya A., "A delay differential equation model for tumor growth" J. Mat. Biol., 47, 270-294, 2003.

[16] Banerjee S., Sarkar R. R., "Delay-induced model for tumor-immune interaction and control of malignant tumor growth" Biosystems, 91, 268-288, 2008. 
[17] Belloma N., Bellouquid A., Delitala M., "Mathematical topics on the modelling complex multicellular systems and tumor immune cells competition" Math. Mod. Meth. Appl. S., 11, 1683-1733, 2004.

[18] Patanarapeelert K., Frank T. D., Tang I. M., "From a cellular automaton model of tumorimmune interactions to its macroscopic dynamical equation: a drift-diffusion data analysis" Math. Comput. Model., 53, 122-130, 2011.

[19] Delitala M., "Critical analysis and perspectives on kinetic (cellular) theory of immune competition” Math. Comput. Model., 35, 63-75, 2002.

[20] Firmani B., Guerri L., Preziosi L., "Tumor/immune system competition with medically induced activation/disactivation” Math. Models Meth. Appl. Sci., 9, 491-512, 1999.

[21] Bellouquid A., Angelis E. De, "From kinetic models of multicellular growing systems to macroscopic biological tissue models" Nonlinear Anal. Real., 12, 1111-1122, 2011.

[22] May R. M., "Biological populations obeying difference equations: stable points, stable cycles and chaos" J. Theoret. Biol., 51, 511-524, 1975.

[23] Gopalsamy K., Liu P., "Persistence and global stability in a population model" J. Math. Anal. Appl., 224, 59-80, 1998.

[24] Muroya Y., Kato Y., “On Gopalsamy and Liu's conjecture for global stability in a population model" J. Comput. Appl. Math., 181, 70-82, 2005.

[25] Uesugi K., Muroya Y., Ishiwata E., "On the global atrractivity for a logistic equation with piesewise constant arguments” J. Math. Anal. Appl., 294, 560-580, 2004.

[26] Muroya Y., "Persistence, contractivityand global stability in logistic equations with piesewise constant delays" J. Math. Anal. Appl., 270, 602-635, 2002.

[27] Muroya Y., "New contractivity condition in a population model with piecewise constant arguments" J. Math. Anal. Appl., 346, 65-81, 2008.

[28] Liu P., Gopalsamy K., "Global stability and chaos in a population model with piecewise constant arguments” Appl. Math.Comput., 101, 63-68, 1999.

[29] Ozturk I., Bozkurt F., Gurcan F., "Stability analysis of a mathematical model in a microcosm with piecewise constant arguments" Math. Biosci., 240, 85-91, 2012.

[30] Gurcan F., Bozkurt F., "Global stability in a population model with piecewise constant arguments” J. Math. Anal. Appl., 360, 334-342, 2009.

[31] Ozturk I., Bozkurt F., "Stability analysis of a population model with piecewise constant arguments” Nonlinear Anal. Real., 12, 1532-1545, 2011.

[32] Bozkurt F., "Modeling a tumor growth with piecewise constant arguments" Discrete Dyn. Nat. Soc., Article ID 841764, 2013.

[33] Li X., Mou C., Niu W., Wang D., "Stability analysis for discrete biological models using algebraic methods" Math. Comput. Sci, 5 (2011) 247-262. 